\title{
Las Capitales Regionales y los Mercado-Santuario de los Toltecas
}

\author{
The Regional Capitals and the Sanctuary-Markets of the Toltecs
}

\author{
Oscar Omaña ${ }^{a}$
}

\begin{abstract}
:
After the fall of Teotihuacan, the Toltec groups from northern Mexico had a rapid expansion throughout Mesoamerica, controlling regions as important as northern and central Veracruz, the Central Highlands, northern Yucatan, and western Mexico, among others. This can only be explained by their effective economic system, with which they maintained tight control over the main sources of raw materials, production centers, trade routes and the most important places of the Epiclassic and Early Postclassic religion. In addition, the model of the "Regional Capitals" allowed them to spread Toltequization throughout the regions in which they were. However, after the fall of Tula, the Toltec's model -the maximum end-, suffered a collapse and the centers most dependent on the capitals experienced an abrupt end, which shows the great dependence of the capitals on the city of Tollan Xicocotitlan and reinforces its role as the center of the world in the Early Postclassic.
\end{abstract}

Keywords:

Toltecs, Early Postclasic, Tula, Huapalcalco, Agricultural Border

\section{Resumen:}

Tras la caída de Teotihuacán, los grupos toltecas procedentes del Norte de México, tuvieron una rápida expansión por toda Mesoamérica, controlando regiones tan importantes como el norte y centro de Veracruz, el Altiplano Central, el Norte de Yucatán, el Occidente de México, entre otras. Esto solo se explica mediante su eficaz sistema económico, con el cual mantenían un férreo control sobre las principales fuentes de materias primas, los centros de producción, las rutas de comercio y los más importantes lugares de la religión del Epiclásico y el Posclásico Temprano. Además, el modelo de las "Capitales Regionales", les permitió difundir la Toltequización por todas las regiones en las que estuvieron. Sin embargo, el máximo fin de los toltecas -difundir su modelo-, tras la caída de Tula, sufrió un colapso y los centros dependientes de la capital experimentaron un abrupto final, lo cual demuestra la gran dependencia de los capitales por la ciudad de Tollan Xicocotitlan y refuerza el papel de ésta, como el centro del mundo en el Posclásico Temprano.

\section{Palabras Clave:}

Toltecas, Posclásico Temprano, Tula, Huapalcalco, Frontera Agrícola

\section{Introducción}

El artículo de Margarita Gaxiola González, "Huapalcalco, un santuario-mercado del Epiclásico en la región de Tulancingo", nos habla sobre una dualidad en cuanto a las funciones del sitio de Huapalcalco, durante el Epiclásico. Sin embargo, al relacionar el carácter que le confirió la autora a otros sitios identificados como toltecas, podemos notar cómo se cumplen algunos de los parámetros propuestos, en cuanto al intercambio y el comercio, haciendo énfasis en el traslado del comercio de las grandes urbes, en este caso Tula, a las ciudadesestado que se encontraban bajo el dominio Tolteca o que formaban parte de sus rutas de intercambio, "incluyendo la participación de las zonas costeras y periféricas" (Gaxiola, 2009: 185). Esto se debió a la continua expansión, hacia las regiones del Centro de Veracruz, Ia Huasteca, la Sierra Norte de Puebla, el Occidente, la Península de Yucatán, la Sierra Madre Oriental y el mismo Altiplano Central, bajo el modelo de la Colonización Deliberada, "en la cual un grupo bien 
definido se desplaza deliberadamente para establecer nuevas comunidades" (Guevara, 2010:103).

Dichas ocupaciones, que en el área actual abarca desde Guanajuato hasta Zacatecas, ayudaron a mantener la Frontera Agrícola o ecológica en el Norte de Mesoamérica o la Mesoamérica Marginal, especialmente "en la segunda etapa de los agricultores en esa área, del 300-900 d.c." (López, 1989:56), hasta el repentino colapso de Tula, que provocó un efecto dominó sobre sus dominios y un abandono total del norte y las migraciones masivas de otras tribus chichimecas, que caracterizaron al final del Posclásico Temprano y los comienzos del Posclásico Tardío.

\section{La expansión militar tolteca}

Los toltecas de Tula, "formados a partir de la integración de los grupos étnicos de los Tolteca-Chichimeca y los Nonoalca" (Jiménez, 1953:14), aquella cultura que entre el final del Epiclásico y el Posclásico Temprano, dominó grandes partes de Mesoamérica, muestra su imponente predominio, ya que "sus conquistas terminaron en Sinaloa por el norte y en la Península de Yucatán por el sureste" (Rivas, 1982: 83). Al pensar en ellos, se nos viene a la mente la imagen de una cultura de guerreros aguerridos, que mediante el uso de su fuerza militar logró extenderse por un amplio territorio, como se ha mencionado. Sin embargo, esto no hubiera sido posible, de no haber establecido el modelo de las Capitales Regionales, el cual es descrito por Sánchez (2018), como cuatro centros principales, todos ellos dependientes de Tula, pero que eran los responsables de abastecer de recursos a la metrópoli, controlar las principales zonas de producción y manufactura de recursos agrícolas, madereros, calizas, minerales, de obsidiana, pesqueros, entre otros. Además, protegían las rutas de comercio, creando de esta forma a otras Tulas Toltecas. La más conocida de estas es Chichén Itzá, "que fue una Tollan o centro civilizador" (Schmidt, 2007:64).

El debate sobre si Chichén Itzá es Tolteca o no, ha generado una cantidad de argumentos entre quienes defienden que lo es y las críticas de quienes no lo hacen. No obstante, entre estos debates nunca se han encontrado presentes aquellas pinturas rupestres, oriundas del municipio de Santo Tomás de los Plátanos, en el Estado de México, las cuales fueron estudiadas por Mateo A. Saldaña a mediados del siglo XX, donde solamente el Museo Arqueológico de Tepeapulco, ha hecho segunda a sus interpretaciones. Estas pinturas son una representación de la conquista de Chichén-Itzá por parte de los toltecas, cuya fecha de elaboración oscila entre el 900 y el 1300 d.c.

Ahora bien, ante la falta de otras evidencias más concretas, me veo en la obligación de darle la razón a
Peter Schmidt, cuando afirmó que: "los Toltecas en Chichén-Itzá es uno de los temas más difíciles de traer en relación con esta antigua capital de los Itzaes en el Norte de Yucatán" (Schmidt, 2007: 64), sobre todo en lo que se refiere al cómo llegaron ahí. Afortunadamente, tenemos a otras dos Tulas, mejor documentadas, TollanChollolan, el lugar de la huida, que de acuerdo con la toponimia, aceptada por el INAH, hace referencia a un segundo grupo de toltecas que llegaron a la región para el año 1000 d.c. Siendo el sitio primeramente habitado por ellos desde el Epiclásico, cuando conquistaron la zona y establecieron un santuario a Quetzalcóatl, "Cholula se organizó alrededor de una administración religiosa del Templo de Quetzalcóatl y estuvo vinculada con el comercio de larga distancia" (Gaxiola, 2009: 187), el cual permaneció hasta el tiempo de los mexicas, siendo un importante centro de comercio.

Finalmente, tenemos a Tollantzingo, "La Pequeña Tula" y en ella encontramos a Huapalcalco. La información que nos brindan los cronistas municipales, recopilada por los Museos de Datos Históricos y de Huapalcalco, en Tulancingo, nos menciona que para el 645 d.c. habrían llegado los toltecas a la zona, cosa que se contradice por completo con el relato de Ce Acatl Topiltzin Quetzalcóatl, que es un personaje con el estatus de Hombre-Dios y que resulta un tema muy complejo por sí mismo.

En los Anales de Cuautitlán, que lo sitúa hacia el siglo IX, y que en conjunto de la Historia Tolteca-Chichimeca, favorecen esta segunda opción, ya que la fecha tentativa para la migración de los Nonoalcas del Golfo es la que más concuerda con este periodo, por lo que es la que acepté para el desarrollo del presente ensayo. Es justo en este sitio donde nos encontramos con la ruta de comercio tolteca mejor reconstruida, aquella que salía de Tula, pasaba por Huapalcalco, para llegar al golfo, la Huasteca, la Sierra Otomí-Tepehua y/o la Sierra Norte de Puebla.

Las cuatro fases del "Imperio Tolteca" están descritas dentro de la sala de exposiciones temporales dedicada a los Toltecas, dentro del Museo Arqueológico de Tepeapulco, dentro del ex-convento de San Francisco. Estas fases se usan para describir los diferentes momentos del desarrollo de la cultura, siendo la primera la Tolteca-Chichimeca. El segundo Imperio está identificado como el Tolteca-Nonoalca y corresponde a Huapalcalco, siendo el "sitio dominante en el Epiclásico" (Gaxiola, 2009:188), el cual se generó con el arribo de Topiltzin Quetzalcóatl y la fusión entre ambos pueblos, una "importancia sagrada de Huapalcalco-Tulancingo" (Gaxiola, 2009:191), que tuvo ya las bases para que fuera considerado como un Santuario para la principal deidad de los toltecas, lo cual muestra el otro lado de la moneda, ya que completa la dualidad del santuariomercado. Sin embargo, no estoy de acuerdo con la 
concepción no militarista que Margarita Gaxiola le da a Huapalcalco, ya que, como se dijo antes, por el Valle de Tulancingo pasaba una de las rutas principales de comercio, además de que se usó como una base para la paulatina expansión tolteca.

Hacia el siglo $X$ d.c., de acuerdo con la información registrada en la enciclopedia de municipios de Hidalgo, que corresponde a San Bartolo Tutotepec, se dio una de estas campañas, cuando los toltecas partieron a la conquista de la Sierra Otomí-Tepehua, importante en cuanto a los recursos madereros y con gran cantidad de fuentes de agua, fundando el sitio de Tutotepec, cohabitando con los Tepehuas. Teniendo este exitoso antecedente, los Toltecas procedieron a incursiones más arriesgadas.

Durante el gobierno de Huemác, el señor de las manos grandes, llegaron a la costa del golfo de México, donde edificaron un templo, del cual creció la actual población de Castillo de Teayo, Veracruz (Rivas, 1982: 84).

Lo anterior fue un punto clave para la creación de la ruta al golfo, que iniciaba en Tollan-Xicocotitlán, pasaba por el Valle de Tulancingo, para después seguir por Toltecatlaxo, "lugar del juego de pelota de los Toltecas" (Acaxochitlán, donde existe un adoratorio de paso teotihuacano con materiales toltecas, reportado por el museo del municipio), cruzando el Patoltecoya "donde pasaron los Toltecas" (río no identificado), para finalmente, llegar a Castillo de Teayo, donde se retoman ciertas ideas de García Payón, en el artículo sobre Castillo de Teayo, realizado por Raúl Flores Guerrero, en donde se afirma que en Teayo, se trasplantó una importante rama de la cultura Tolteca, que todavía no ha sido identificada. Aunado a lo anterior, podemos notar que el sitio "tiene un estilo Huasteco, influenciado por la cultura Tolteca y la cultura Azteca" (Flores: 1958:6). Finalmente, se puede afirmar que de la Huasteca veracruzana, los Toltecas habrían descendido al centro y sur de Veracruz, como demuestran los materiales toltecas en el sitio de Quiahuiztlán, los que atestiguan una invasión y conquista por parte de ellos.

\section{Comercio y Tributo: Los Mercados-Santuario}

Una vez que se concluyeron las conquistas hacia el golfo, se establecieron las rutas de comercio, ahora protegidas por las colonias o asentamientos toltecas. Aquellos sitios que participaron en la ruta del golfo pudieron estar identificados por lo que Margarita Gaxiola describe como un sistema de los yugos, para identificar a los sitios en una red de comercio determinada, dado que tres de estos se han encontrado en Huapalcalco, todos de piedra verde.

Gaxiola tiene la teoría sustentada en el encuentro de una cantidad considerable de yugos de este tipo, que van desde San Luis Potosí hasta el Centro de Veracruz. Tampoco debemos olvidar que Huapalcalco fue un importante centro de extracción y manufactura de la obsidiana, tanto para los Teotihuacanos como para los Toltecas, "desde la perspectiva arqueológica es importante conocer cómo la ciudad de Tula y su esfera, desarrollaron un suministro regular de obsidiana a partir de varios yacimientos" (Pastrana, 2010: 62). Uno de estos, fue el yacimiento del Pizarrín, cuya explotación está descrita como "una producción destinada a satisfacer las necesidades de consumo por medio del intercambio mercantil" (Gaxiola, 2009: 191), no siendo la única fuente de obsidiana, ya que se explotó la Sierra de las Navajas por igual, además de muchos otros lu gares, pero estos dos ejemplos son los más comprobados.

...la explotación de la obsidiana verde del yacimiento de

Sierra de las Navajas por el estado Tolteca, se relaciona con la forma de control del territorio y de una fuente de materia prima básica para el desarrollo de la sociedad en múltiples aspectos (Pastrana, 2010:62).

Es probable pensar que tanto el Pizarrín como el Cerro de las Navajas aportaban "un suministro de núcleos, raspadores y excéntricos" (Pastrana, 2010:67). Eso sin mencionar la autosustentabilidad del asentamiento de Huapalcalco, pues existieron una gran cantidad de terrazas agrícolas por todo el Valle, que abastecían de alimentos y de la misma forma, de la obsidiana, y que se comerciaba con los excedentes.

Para procurar el buen funcionamiento de las rutas de comercio y constante suministro de todo tipo de recursos, se tuvieron que reforzar los caminos que iban a Tollan, destacando el dato proporcionado por Sánchez en el año 2018, en el que menciona que la cantidad de provincias tributarias que tuvo Tula y su Triple Alianza sobrepasaban las 100 .

Aunado a lo anterior, "en la Fase de apogeo Tula mantuvo interacciones económicas, incluso control político sobre el bajío al noroeste de Tula" (Pastrana, 2010:61), principalmente por las amenazas de los nómadas, quienes en el Posclásico Tardío asolaban esas regiones, pues sitios como Actopan o Ixmiquilpan eran considerados como la frontera con los Chichimecas. Por ello se conquistaron los actuales municipios de Nopala de Villagrán, colindante con Querétaro y que pudo haber pertenecido a la jurisdicción de El Cerrito, una capital regional Tolteca, que controlaba el sureste del estado y la región colindante con Hidalgo, de la cual obtenía sus tributos, y servía como paso hacia Guanajuato y los asentamientos del norte, en los límites de la frontera agrícola o al Occidente. Además, fungía como un santuario "del culto a Quetzalcóatl, la Serpiente Emplumada, Venus o Ehécatl" (Gaxiola, 2009:186). Inclusive, hasta el siglo XVII, seguía sirviendo como santuario, cuando los indígenas de la Ciudad de Querétaro iban a llevar sus ofrendas a la pirámide que apodaron como "El Pueblito". 
El dominio del Norte de la Cuenca de México fue otra de las prioridades, Miguel Guevara señala que "fue un control tardío de esa región para el final de la Fase Tollan (1000-1200 d.c.)" (Guevara, 2010:103). Gracias a este control de la zona se pudieron establecer cuatro cabeceras: la primera, en Apaxco, para tener el control de las calizas de esa área. Cabe señalar la existencia de otras calizas en la región de Tula, las cuales también fueron explotadas para la fabricación del estuco. La segunda, en El Pedregal, donde tuvieron acceso al tezontle de esa zona. La tercera, en el pie de monte de la Ahumada, mientras que la última fue "el complejo urbano San Miguel Eyalco-Santa María, que controlaba a los llanos de Pachuca y el principal asentamiento de Tizayuca" (Guevara, 2010:104).

Debido a lo anterior, la zona de Huey Poxtla-Atotonilco tuvo "una pronunciada nucleación de la población y centralización del poder" (Guevara, 2010:105), la toma de Tlahuelilpan, "en donde se riegan las aguas", se dio para aprovechar sus recursos agrícolas y suelos fértiles de abundantes cosechas, que caracterizan a la zona y siguen siendo uno de sus motores económicos actuales, como lo demuestran los datos estadísticos del municipio. Por último, se logró el control sobre la zona de San Agustín Tlaxiaca, "lugar del juego de pelota", donde se construyeron adoratorios de paso, una especie de pequeños basamentos donde los viajeros podían descansar y adorar a las deidades de los mercaderes, Quetzalcóatl o cualquier otra a la que se venerase. Se sabe de la existencia de cuando menos uno de estos en la comunidad del Chamizal, dentro del poblado de San Juan Solís, por un reporte que hicieron los pobladores al Centro INAH-Hidalgo, para saber si aquella construcción interfería con sus labores de cultivo. Por lo que se hizo una rápida excavación y salvamento, recuperando una escultura antropomorfa completa, identificada como un portaestandarte y la cabeza antropomorfa de una segunda, además de material cerámico, identificado como Tolteca y Azteca. No obstante, se dio el visto bueno para que fuera destruida, pero sirvió como una evidencia concreta para demostrar la ocupación Tolteca en el área.

\section{La Frontera Agrícola o Ecológica: Del Clásico a la Caída de Tula}

Pero a todo esto, ¿qué sucedía con la frontera ecológica o agrícola? y ¿cuál era su importancia? Volvamos al pasado, para poder entender de mejor forma su contexto, remontémonos a cerca del año 500 d.c., cuando se da "la incorporación de la Cultura Chalchihuites al vasto sistema de intercambio mesoamericano" (López, 1989:57), como consecuencia del poblamiento que se estaba dando, impulsado por Teotihuacán, para aprovechar los recursos mineros de la zona de Zacatecas y establecer una ruta de comercio con Aridoamérica, y generando con ello, "el su rgimiento de un gran centro hegemónico La Quemada" (López, 1989:57). Este último, en su momento de mayor esplendor, tuvo unas 17 hectáreas de extensión, con una población superior a cualquier otro asentamiento de la zona, solo siendo comparada con Altavista, un centro fundado tiempo antes, o Paquimé.

Aunado a lo anterior, tenemos que "La Quemada fungió como un puerto de intercambio en una ruta de comercio continental que enlazaba Teotihuacán, con Paquimé y Chaco Canyon" (López, 1989:59); servía además, como un centro donde se concentraban los recursos mineros provenientes de la "extracción de metales, turquesa y minerales" (López, 1989: 58). Zacatecas quedaba como el límite de la frontera agrícola, donde se localizaban los últimos puntos poblados por los mesoamericanos, sostenida por los teotihuacanos, quienes establecieron una serie de colonias en esta zona:

...las funciones de estas colonias gobernadas por burócratas Teotihuacanos o Toltecas, quedarían reducidas a la contención de los embates de los nómadas hacia el sur y el aprovisionamiento de recursos exóticos, Altavista a Teotihuacán y La Quemada a Tula" (López, 1989: 60).

Estoy un tanto en desacuerdo con esto último, ya que si bien esas eran sus labores principales, no debemos olvidar la importancia que éstas tuvieron en el comercio con Aridoamérica y la colonización del norte, la cual no se terminó de dar, ya que con el colapso de Teotihuacán, sumado a una serie de cambios climáticos, acabaron con esta frontera agrícola, aunque esta siguió poblada por agricultores de la Cultura Chalchihuites hasta el Posclásico Temprano.

De acuerdo con Leonardo López Luján, los agricultores antes mencionados tuvieron relaciones con los toltecas, pero éstas no fueron tan grandes como las que habrían tenido con los teotihuacanos, debido a que estos no colonizaron más allá del estado de Guanajuato en esta parte de Mesoamérica. Nunca se adentraron del todo a la Gran Chichimeca, generando migraciones masivas hacia el Altiplano Central, en donde los ToltecasChichimecas formaban parte de una de estas migraciones, y he aquí donde radica la importancia del sitio de Cañada de la Virgen, San Miguel de Allende, Guanajuato, que junto con la Quemada son consideradas dentro del esquema de los cuatro Imperios o fases del Imperio Tolteca, como las primeras sedes de éste, y donde se formó la nueva frontera agrícola 0 ecológica de los toltecas.

Cañada de la Virgen o la Casa de los Trece Cielos, "se encuentra ubicada en el límite de la frontera de Mesoamérica" (Quiroz, 2019:1). Era una capital regional Tolteca, que servía como el límite de la frontera agrícola, sostenida agrícolamente por su cercanía con el río lajas 
y por el constante envío de recursos por parte del Altiplano Central. Pero si solo la describo de esa manera, sería restarle su debida importancia, ya que este sitio fue, de acuerdo con Rossana Quiroz, donde se desarrolló el calendario agrícola usado por los toltecas:

...el basamento del conjunto central, como un instrumento calendárico y astronómico capaz de capturar las posiciones del Sol, la Luna y Venus, a través de los vértices que componen sus cuerpos escalonados (Quiroz, 2019: 2).

Su principal ocupación se dio entre el 540-1050 d.c., "en el siglo X los niveles pluviales que sostenían a los grupos agrícolas descien den" (Quiroz, 2019: 8), nuevamente los cambios climáticos y el agotamiento de los recursos les jugaron en contra a los habitantes de la frontera agrícola y a los pocos habitantes de Chalchihuites que aún quedaban en Zacatecas y quienes dependían de sus parientes toltecas. Con este segundo colapso de la frontera agrícola, las invasiones de los chichimecas y otras tribus del norte, comenzaron y tuvieron un amplio alcance, inclusive la misma capital tolteca.

Tollan, el centro del mundo mesoamericano del Posclásico Temprano, fue incendiada cayendo ante los invasores del norte y las rebeliones internas que se produjeron, debido a un modelo de integración social que nunca terminó de encajar entre las culturas a las que dominaron, como a los otomíes, los tepehuas, toton acas y otros grupos chichimecas. No pasó mucho para que el territorio de la Gran Chichimeca abandonara su agricultura y volviera a una subsistencia nómada, tipo cazador-recolector; "hacia el 1100 d.c. se da el final ocupación de los pueblos agricultores en las zonas central y noroccidental de la Mesoamérica Marginal" (López, 1989:79), esta debacle no solo se dio en el norte y en la capital Tolteca, los centros provinciales su frieron de un efecto dominó, una vez que se vio perdida su capital. En el caso de Huey Poxtla-Atotonilco, "los materiales recuperados en el sitio indican un abandono súbito, ya que se recuperaron materiales completos en contextos domésticos" (Guevara, 2010:105).

Toda la evidencia indica que el colapso Tolteca fue un evento inesperado, un suceso repentino, que como consecuencia produjo grandes cambios, primero en el Altiplano Central y después, en todas las zonas que estos controlaron. Algunas de sus capitales o cabeceras regionales se salvaron, como ejemplo tenemos a Cholula, Huapalcalco o el Cerrito, curiosamente estas tres gozaban de un carácter sacro-religioso que se acrecentó con el tiempo, otras más fueron ocupadas por los antiguos grupos nativos o invasores, como Quiahuiztlán por los Totonacas, Tutotepec por los Otomíes, Tenayuca por los Chichimecas de Xólotl o Castillo de Teayo por los Huastecos, mientras que una gran cantidad de sus asentamientos se abandonaron totalmente, ni siquiera la Tollan de la Península de Yucatán sobrevivió mucho tiempo a la caída de Tula.

\section{Conclusiones}

El modelo desarrollado por los toltecas, de las Capitales Regionales, es un modelo económico fácilmente reconocible, en donde existen áreas ricas en recursos naturales o que manufacturan bienes preciados, que están sujetas a una ciudad-estado que las controla, tanto a ellas como a su producción, para poder abastecerse a sí misma, y enviar los excedentes hacia el así considerado por Sánchez (2019), centro del mundo durante el Epiclásico y el Posclásico temprano: TollanXicocotitlán, a la vez que estas capitales se encontraban estrechamente vinculadas con alguna ruta de comercio importante o que las conectaba por completo, salvo el caso de Chichén-Itzá, que genera más dudas que respuestas sobre el tipo de relación que tuvo con los Toltecas. Sin embargo, quedarnos con que solo era un modelo económico sería restarle importancia a estas "Capitales Regionales", pues de igual forma sirvieron como centros de la toltequización, una forma en que las sociedades chichimecas, otomíes, totonacas, itzaes, huastecas, tepehuas, entre muchas otras, abandonaban sus costumbres y adoptaban las Toltecas, teniendo un mejor éxito en ciertas partes o ámbitos.

Un ejemplo de lo anterior es el culto a Quetzalcóatl, en su advocación de Ehécatl, que fue un éxito entre los huastecos, recordando que los toltecas buscaron homogeneizar a Mesoamérica, siendo este uno de los principales motivos de su rápida expansión y repentina caída, ya que nunca terminó de cuajar este modelo. Además, con la caída de la Frontera Agrícola, todo esto se aceleró aún más, abandonando a la agricultura, dejando a un lado la mayoría de los conocimientos mesoamericanos, que databan de la época de la colonización teotihuacana y la subsecuente, pero menor, ocupación Tolteca.

El caso del trabajo realizado por Leonardo López Luján en el estado de Zacatecas, sirve para entender a las complejas sociedades del norte, sobrepasando el estigma de bárbaros, en el que se tenía a los Chichimecas, término despectivo empleado por los Mexicas para referirse a los grupos nómadas del norte, mismos que en su época imperial pregonaban de su supuesta Toltecayótl, sin recordar o saber que en algún momento de la historia de ambas culturas, estos pertenecieron a los grupos Chichimecas.

\section{Referencias}

Catálogo de zonas arqueológicas del INAH, Zona arqueológica de Cañada de la Virgen. Disponible en: https://inah.gob.mx/zonas/79-zonaa rqueologica-canada-de-la-virgen, consultada: 5 de junio de 2020. 
Catálogo de zonas arqueológicas del INAH, Zona arqueológica de Cholula. Disponible en línea: https://inah.gob.mx/zonas/12-zonaarqueologica-de-cholula, consulta da el 12 de junio de 2020-06-12

Catálogo de zonas arqueológicas del INAH, Zona arqueológica de El Cerrito, Disponible en línea: https://inah.gob.mx/zonas/122-zonaarqueologica-el-cerrito ], consultada: 5 de junio de 2020

Catálogo de zonas arqueológicas del INAH, Zona arqueológica de Quiahuiztlán, Disponible en línea https://inah.gob.mx/zonas/165-zonaarqueologica-el-cerrito, consultada: 5 de junio de 2020 .

Enciclopedia de Municipios del Estado de Hidalgo, Nopala de Villagrán, https://intranet.e-hidalgo.gob.mx/enciclomuni/municipios/13044a.htm, consultada: 12 de junio de 2020.

Enciclopedia de Municipios del Estado de Hidalgo, San Agustín Tlaxiaca, Disponible en línea:https://intranet.ehidalgo.gob.mx/enciclomuni/municipios/13052a.htm, consultada: 12 de junio de 2020 .

Enciclopedia de Municipios del Estado de Hidalgo, San Bartolo Tutotepec. Disponible en línea: https://intranet.ehidalgo.gob.mx/enciclomuni/municipios/13053a.htm, consultada: $5 \mathrm{de}$ junio de 2020.

Enciclopedia de Municipios del Estado de Hidalgo, Tlahuelilpan, en línea https://intranet.e-hidalgo.gob.mx/enciclomuni/municipios/13070a.htm, consultada: 12 de junio de 2020.

Enciclopedia de Municipios del Estado de Hidalgo, Tulancingo de Bravo, Disponible en línea: https://intranet.ehidalgo.gob.mx/enciclomuni/municipios/13077a.htm, consultada: 12 de junio de 2020.

Enciclopedia de Municipios del Estado de México, Santo Tomás de los Plátanos, Disponible en línea https://infed.gob.mx/work/enciclopedia/EMM15mexico/municipios/15 078 a html, consultada: 5 de junio de 2020 .

Flores, Raúl (1958) "Castillo de Teayo" Anales del Instituto de Investigaciones Estéticas. Vol. 7, No. 27. UNAM, pp. 5-15

Gaxiola, Margarita (2009) "Huapalcalco, un santuario-mercado del Epiclásico en la región de Tulancingo". Caminos y mercados de México, UNAM, pp. 185-220

Guevara, Miguel (2010) "La caída de los centros provinciales Toltecas. El caso de Atotonilco de Tula". Estudios de Antropología e Historia. Arqueología y Patrimonio en el Estado de Hidalgo. No. 8. UAEH, pp. 101-122

López Luján, Leonardo (1989) Nómadas y Sedentarios. El pasado Prehispánico de Zacatecas, México, INAH.

Museo Arqueológico de Acaxochitlán.

Museo Arqueológico de Tepeapulco.

Museo de Datos Históricos de Tulancingo.

Museo de Huapalcalco.

Pastrana, Alejandro (2010) "La secuencia de explotación de la obsidiana de la Sierra de las Navajas, Hidalgo, México". Estudios de Antropología e Historia. Arqueología y Patrimonio en el Estado de Hidalgo, No. 8. UAEH, pp. 55-84

Quiroz, Rossana (2019) “El Basamento Piramidal de Cañada de la Virgen como Calendario del Horizonte Artificial". Revista Chicomoztoc. Vol. 2, No. 2, UAZ

Rivas Paniagua, Enrique (1982) "Hidalgo, entre selvas y milpas... la neblina”. Monografía Estatal, SEP,

Schmidt, Peter J. (2007) “Los 'Toltecas' de Chichén Itzá, Yucatán”. Arqueología Mexicana. No. 85, mayo-junio, Raíces, pp.64-68

Sánchez Vázquez, Sergio (2018) “Tollan Xicocotitlán y su señor Quetzalcóatl: El proceso de constitución del 'centro del mundo' durante el Posclásico Temprano”. Tula y su Jurisdicción Arqueología e Historia. UAEH, pp. 11-42. 